\title{
$1 \quad$ Rethinking alkylating(-like) agents for solid tumor management
}

2 Hélène Lajous ${ }^{\mathrm{a}, \mathrm{b}}$, Bénédicte Lelièvre ${ }^{\mathrm{c}}$, Elodie Vauléon ${ }^{\mathrm{d}, \mathrm{e}}$, Philippe Lecomte $^{\mathrm{b}, *}$ and 3 Emmanuel Garcion ${ }^{\mathrm{a}, *}$

$4 \quad{ }^{a}$ CRCINA, INSERM, Université de Nantes, Université d’Angers, Angers, France

$5 \quad{ }^{\mathrm{b}}$ Center for Education and Research on Macromolecules (CERM), CESAM Research Unit, University 6 of Liege, B6a Sart-Tilman, B-4000 Liege, Belgium

$7 \quad{ }^{\mathrm{c}}$ Centre régional de pharmacovigilance, Laboratoire de pharmacologie-toxicologie, CHU Angers, 4 rue 8 Larrey, F-49100 Angers, France

$9 \quad{ }^{\mathrm{d}}$ Centre Eugène Marquis, Rennes, France

10 e INSERM U1242, Université de Rennes 1, Rennes, France

$11 *$ Equivalent contribution

12 Correspondence: emmanuel.garcion@univ-angers.fr (E. Garcion) 


\section{Abstract}

Although old molecules, alkylating agents and platinum derivatives are still widely used in the treatment of various solid tumors. However, systemic toxicity and cellular resistance mechanisms impede their efficacy. Innovative strategies, including local administration, optimization of treatment schedule/dosage, synergistic combinations and encapsulation of bioactive molecules within smart multifunctional drug delivery systems, have shown promising results to potentiate anticancer activity while circumventing such hurdles. Furthermore, questioning the old paradigm according to which nuclear DNA is the critical target of their anticancer activity has shed light upon subcellular alternative and neglected targets that obviously participate in mediating cytotoxicity or resistance. Thus, rethinking the use of these pivotal antineoplastic agents appears critical to improve clinical outcomes in the management of solid tumors.

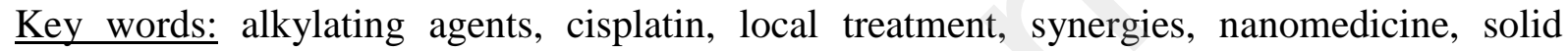
tumors 


\section{Strategic paths towards anticancer therapy}

Oncology mainly focuses on patient symptoms, treating hallmarks acquired by normal cells that gradually progress to a neoplastic state, instead of fighting against a still unknown causal entity responsible for cancer occurrence and progression [1]. Global strategies, namely chemotherapy and radiotherapy, still consist in the mainstay of the treatment of solid tumors by addressing specific mechanisms involved in tumorigenesis. More targeted therapies (see Glossary), such as anti-angiogenesis strategies, have been developed with various degrees of success depending on the patient pathogenesis [2,3]. A better insight into the diverse underlying processes, including causes, triggered cellular and molecular pathways and potential related targets, would definitely help for developing relevant and effective anticancer treatments.

In contrast to the empiricism from animal models that gave rise to alkylating agents or to the rational design emanating from the targeting of pathways altered in tumors, we suggest that rethinking the use of conventional anticancer drugs could make it possible to exploit their full potential. This alternative approach relies on the optimization of an already marketed bioactive drug capable of reaching its target in effective concentrations for exerting its anticancer activity while limiting adverse side effects. In this context, alkylating agents are old molecules still widely used in the front-line treatment of various solid tumors. Among them, platinum derivatives do not alkylate but rather complex with their nucleophilic targets. Although historically affiliated to alkylating agents, they should therefore rather be referred to as "alkylating-like” agents. Half of patients experience platinum-based drug therapy [4,5]. As such, the clinical relevance of platinum compounds is key in the daily practice. Cisplatin is the oldest platinum drug approved by the FDA. Although alternative platinum derivatives have been developed to improve its therapeutic index, cisplatin remains the leader molecule of platinum complexes and one of the most compelling anticancer drugs with a pivotal role in the management of solid tumors [6,7]. Therefore, cisplatin will be addressed as a prototypic platinum-based anticancer agent to exemplify paradigms, mechanisms, limitations and new directions that fall under a broader understanding of the future of alkylating agents and platinum compounds in the clinic.

In the following, we provide an up-to-date review of the rationale and conventional use of alkylating agents and platinum derivatives in clinical practice. Then, we focus on optimization ways, synergies and innovative alternatives that pave the way for rethinking how 
to potentiate their anticancer efficacy, laying down future challenges for these old molecules in the treatment of solid tumors, with the ultimate view of personalized medicine.

\section{Rationale and conventional use of alkylating agents and platinum derivatives in clinical practice}

After the attack of Bari Harbor in 1943 revealed the effects of mustard gas on bone marrow depletion and first therapeutic outcomes on lymphoma, alkylating agents gradually became a gold-standard as first-line treatment in various cancer indications. The DrugBank database reports all FDA-approved alkylating agents and affiliated compounds in worldwide use, their initial indications, delivery type and administration route (Table 1) [8]. Other alkylating agents (e.g. mitolactol that has been granted orphan drug designation from the FDA for the treatment of invasive carcinoma of the uterine cervix and as adjuvant therapy in the treatment of primary brain tumors) and platinum complexes (lobaplatin for inoperable metastatic breast cancer, chronic myelogenous leukemia and small cell lung cancer in China, heptaplatin for gastric cancer in Korea, nedaplatin for (non-)small cell lung cancer, esophageal cancer and head and neck cancer, and miriplatin for hepatocellular carcinoma in Japan) are also currently in use in humans [5].

\section{Mechanism}

Anticancer agents are traditionally classified in chemical families according to their mode of action. Intercalating and alkylating agents are reported to directly interact with DNA by inter- or intrastrand crosslinking. However, the mechanism of action of intercalating agents that form formaldehyde-based covalent bonds with DNA bases as shown through the example of anthracycline antibiotics on Figure 1-(a) strictly differs from that of alkylating agents [9]. Alkylators allow for the transfer of an alkyl group from one to another molecule under physiological conditions. Such nucleophilic substitutions occur by an $S_{N} 1$ or $S_{N} 2$ mechanism depending on the kinetics of the reaction and result in covalent binding to an organic macromolecule as depicted in Figure 1-(b) in the case of temozolomide [10]. Since exposure to alkylating agents leads to chromosomal aberrations in dividing cells, DNA stands for the key target site of alkylation within cells. This hypothesis is further supported by its high molar mass, which makes DNA the major nucleophilic substrate for alkylation within the organism, far ahead RNA and proteins. Alkylation mainly occurs during $\mathrm{S}$ phase, while DNA is 
replicating: both strands are separated making nucleophilic substrates easily reachable. A blockage in G2-phase was also reported [11]. Alkylating agents are more likely to bind to exposed nucleophilic sites in the grooves of the DNA double helix: guanine (positions $\mathrm{N}_{7}, \mathrm{O}_{6}$, $\mathrm{N}_{2}$ and $\mathrm{N}_{3}$ ), adenine $\left(\mathrm{N}_{3}\right.$ and $\left.\mathrm{N}_{7}\right)$ and cytosine bases $\left(\mathrm{N}_{3}\right)$. The resulting adducts prevent strands from uncoiling and separating, making DNA replication and downstream RNA transcription impossible where the alkylation occurred. Platinum complexes are stabilized by various ligands that can be substituted by nucleophilic substrates to form a strong coordination bound with the central platinum atom. In this respect, platinum compounds were historically considered as alkylating agents even though they do not interact with biological macromolecules through an alkyl group but rather by complexation. Cisplatin, whose mechanism of action is illustrated on Figure 1-(c), enabled to dramatically improve the prognosis of germinal cancer cells and is still currently used as a gold-standard in the treatment of various solid tumors [6,7]. Contrary to alterations caused by mono-functional alkylating agents such as nitrosoureas, inter- or intracatenary bridges induced by platinum derivatives between both DNA strands are extremely difficult to repair.

\section{Resistance}

Intrinsic or acquired resistance to alkylating agents and platinum derivatives is considered as a multifactorial phenomenon. In the case of cisplatin, it involves avoidance (e.g. drug exclusion from the cell [12-14] or from the nucleus [15]), prevention or escape (e.g. drug inactivation [4,6,15-17] or resistance to apoptosis [11,13,14,18-20]) and repair (e.g. DNA repair [6,13,15,16,21-24]) mechanisms. Multiple intrinsic regulators that may also be modulated by extracellular triggers represent key (in)activators of these pleiotropic processes, as exemplified by mTOR in autophagy or microRNAs (miRNA), and could be identified as relevant predictive biomarkers of patient response to a treatment with the perspective of providing more accurate and personalized chemotherapeutic regimen [24]. Figure 2-(a), Key Figure, illustrates the main cellular mechanisms that mediate resistance to cisplatin. The development of alternative platinum derivatives with a milder toxicity profile and able to alter all cells whatever their stage in the cell cycle, including stem cells located within the tumor margins that are insensitive to radio- or chemotherapy, is of particular interest to circumvent drug resistance [6]. 


\section{Radiosensitization}

Radiotherapy (RT) constitutes a key strategy in the treatment of several solid tumors, including glioma, lung, breast, head and neck, uterine cervix, rectum, vulvar and prostate cancers. Radiation beam causes direct DNA damages but also indirectly impact cell-death through the formation of highly reactive oxygen species (ROS). Modulation of the tumor response to RT can be achieved by resorting to various antineoplastic agents and has been extensively investigated in alkylating agents and platinum-based strategies with the aim of amplifying the differential effects between tumor and normal cells [25-28]. Due to their ability to form DNA adducts leading to double-strand breaks and the heavy platinum atom that locally enhances the effect of external beam radiation respectively, alkylating agents and platinum compounds are particularly used in combination with RT as effective radiosensitizing chemotherapy [25,27-29]. Clinical studies have further evidenced the superior efficacy of concomitant chemoradiotherapy in various solid tumors compared to RT alone $[25,26,30]$. This synergistic effect depicted in Figure 2-(b) can be explained by a more accurate locoregional control of the pathology with a reduced or at least contained tumor cell proliferation that would otherwise quickly entails radioresistance, resulting in a better prognosis. Paradoxically, radioresistance can also occur from disruption of blood supply to the altered tissue after surgery and chemotherapy, leading to hypoxic foci. Tumor radiosensitivity can then be modulated by chemical radiosensitizers that simultaneously enhance the therapeutic benefit of RT locally and exert their own cytotoxic effect [31,32]. The time schedule between chemotherapy and RT is a key point for effective combination owing to dose- and time-dependent cytotoxicity of the drug, leading to synergism or at least to an additive effect on tumor cells [30]. Polychemotherapy, i.e. the combination of several drugs, offers another way to reach synergism in anticancer treatment.

\section{Polychemotherapy}

Alkylating agents as well as platinum compounds are commonly used concurrently with other antineoplastic agents, including targeted drugs and antibodies, in the management of solid tumors. The combination of drugs that exert their anticancer activity through various mechanisms of action induces cell damage and metabolism dysfunction by altering several molecular targets and signaling pathways involved in tumorigenesis [14,33]. This option is therefore commonly considered in clinical practice to potentiate drug efficacy and reverse acquired drug resistance like in ovarian, biliary tract, lung, breast and prostate cancers that 
primary respond to a platinum-based treatment but ultimately relapse. For instance, the standard treatment for patients with advanced colorectal cancer that consists of the combination of 5-fluorouracil, leucovorin and oxaliplatin, demonstrated a potentiation of the anticancer activity of oxaliplatin with fluoropyrimidines resulting in a significant improvement in overall survival. The design of complementary targeted drugs since the 2000s has further reinforced this trend [34,35]. In parallel, in the mid-1970s, a breakthrough in the treatment of men with metastatic testicular cancer arose from a combinatory regimen based on cisplatin supplemented with bleomycin and vinblastine, leading to an increase in complete response rates from $5 \%$ to $60 \%$. Substitution of vinblastine with etoposide further enabled to reach up to $80 \%$ of cure rates [6]. Additional adjunctive drugs can also be considered to modulate platinum activity or toxicity [36-41].

\section{Optimization of the use of alkylating agents and platinum derivatives}

Although dramatically limited by resistance mechanisms and a lack of specificity associated with high systemic toxicity, alkylating agents and platinum derivatives remain pivotal in the management of solid tumors. Promising alternatives to their conventional use in clinical practice will be addressed in the following part that paves the way for reflection on an optimization of their use in anticancer therapy and suggests that time may have come to bring these old molecules back on the stage again.

\section{Drug administration and dosage}

Chemotherapy is often limited by systemic injection which causes drug dilution within the organism and is responsible for severe side effects, especially on highly proliferative cells. High systemic toxicity of conventional anticancer agents can be overcome by using a more suited route of administration depending on the tumor type. In the case of operable patients with glioblastoma, an alternative to temozolomide relies on the implantation of carmustineloaded wafers (Gliadel ${ }^{\circledR}$ ) within the resection cavity at the end of the surgery [42,43]. In such aqueous environment, the anhydride bonds of the biodegradable polymeric matrix get hydrolyzed, allowing for a controlled and sustained release of the drug that can diffuse within the surrounding parenchyma during several weeks. After degradation, the active metabolite can alkylate DNA, cross-link with RNA and entail proteins carbamylation, ultimately leading to cell apoptosis [43]. Although Gliadel ${ }^{\circledR}$ demonstrated a high therapeutic efficacy in animal models, clinical translation is limited by side effects and poor diffusion within the damaged 
parenchyma since the concentration gradient may not be strong enough to allow carmustine for penetrating deep into the brain tissue and for distributing through the tumor margins [4447]. Although alkylating agents have provided therapeutic efficacy and improved patient outcomes in the management of brain cancer, alternative strategies are required to reach therapeutic doses in close vicinity of the tumor burden and maximize their anticancer activity.

In this context, the locoregional administration of chemotherapy directly within the brain enables both to bypass the blood brain barrier that prevents most macromolecules and therapeutic drugs from reaching the central nervous system and to locally increase drug concentration. Convection-enhanced delivery (CED) consists of infusing the drug at high concentration directly within the brain or the tumor via intraparenchymal microcatheters [44]. A constant hydrostatic positive pressure gradient is established by an infusion pump that forces convection of the therapeutic solution at a rate of 0.1 to $10 \mu \mathrm{min}^{-1}$. As such, CED achieves homogeneous elliptical to spherical distribution of molecules of various molar masses over large distances compared to suboptimal therapeutic doses reached by passive diffusion from concentration gradient [45,48-50]. Because they cannot easily cross the blood brain barrier, platinum derivatives do not reach brain tumors in optimal therapeutic concentrations when administered intravenously [51]. In animal models, CED was shown to dramatically increase the concentration of cisplatin and carboplatin within the brain tumor in regard with traditional administration routes while reducing systemic toxicity [52]. Although safety and feasibility have been demonstrated in phase I clinical trials, translation to the clinics failed so far because of surgical complications [53,54]. In addition, increased interstitial fluid pressure within brain tumors and leakage into the cerebrospinal fluid drastically reduce drug concentration at the targeted site and can even induce neurotoxicity $[55,56]$. Thus, technical advances are expected to fill the gap between the view of CED as a promising strategy to deliver therapeutic agents in situ to large and clinically-relevant brain volumes and the current state of an invasive technique in which continuous or repeated administration is at risk due to infection, hemorrhage or neurologic disorders related to catheter positioning inside the brain parenchyma [45,55,57]. In case of localized diseases, other clinically-relevant routes of administration have been investigated such as intraperitoneal chemotherapy for primary or recurrent ovarian cancer $[58,59]$.

The use of drugs at their maximum tolerated dose (MTD) requires intermittent drugfree periods between two cycles of chemotherapy that should allow the patient for recovering from acute toxicities. However, tumor cells can regenerate during that resting time, and 
selected clones may develop resistance to the treatment [60,61]. As a result, the traditional rationale according to which higher doses are necessary for tumor eradication is slowly shifting to the concept that "less is more", which favors a stabilization of the disease over time for maintenance of quality of life. As hyperfractionated radiotherapy suggests that a continuous low-dose schedule may be more efficient in killing highly-proliferative cells than standard radiotherapy by avoiding tumor cells reparation, metronomic chemotherapy consists in the chronic and equally-spaced administration of drugs at low dose $\left(1 / 10^{\text {th }}\right.$ to $1 / 3^{\text {rd }}$ of the MTD) without extended rest periods [33,62]. Whereas drug administration by intermittent bolus generally results in high peak plasma concentrations that are further responsible for severe toxicity, “dose-dense" strategies have shown encouraging results with evidence of disease stabilization and improved outcomes associated with a low toxicity profile in patients with solid tumors [61,63-65]. Interestingly, the frequent low-dose administration of traditional drugs makes them able to target the dividing vascular endothelial cells, thus demonstrating additional anti-angiogenic potential, while the stimulation of the anticancer immune response may further contribute to force tumor dormancy $[33,60,62,64]$. Besides, metronomic chemotherapy results in more convenient treatment administration and promotes maintenance of patients quality of life [65]. Economic reasons can also favor oral metronomic chemotherapy as a minimal cost but still compelling alternative to current standard-of-care, particularly in developing countries [66,67]. Metronomic regimens based on alkylating agents or platinum derivatives have demonstrated a therapeutic benefit in patients with solid tumors $[60,65,68]$. However, large-scale studies and controlled randomized trials that compare conventional MTD to the same metronomic administration regimen are required to define the optimal drug dosage and schedule.

\section{Alternative and neglected targets}

Since chromosomal aberrations in dividing cells were an outstanding feature of mustard gas intoxication, most hypotheses postulated that nuclear DNA was the most critical pharmacological target of alkylating agents and platinum derivatives [13,14]. However, in the case of platinum-based treatments, the level of Pt-DNA adducts does not necessarily correlate with neither intracellular drug accumulation nor cytotoxicity, suggesting that other cellular or molecular components must be involved with various degrees of specificity and severity in anticancer activity [4,16,25,69,70]. Growing evidence notably suggest the role of mitochondria in cisplatin anticancer activity [13,17,71,72]. Mitochondria are involved in the apoptotic pathway through the release of cytochrome c into the cytosol and subsequent 
activation of caspases 8 and 9, thus constituting a critical target for cytotoxic drugs. Rerouting chlorambucil through engineered mitochondria-penetrating peptides (MPPs) that are able to cross the dense and highly hydrophobic membranes of mitochondria demonstrated a dramatic potentiation of its anticancer activity in various cancer cell lines by promoting apoptosis and evading deactivation processes that commonly occur within the cytosol [73]. Interestingly, the development of a cisplatin analog from MPPs showed that mtDNA damage was sufficient to induce cytotoxicity and promote apoptotic cell death without impairing nuclear DNA or entailing cell cycle arrest [74]. Therefore, mitochondria-specific targeting should be reconsidered for implementing innovative and efficient anticancer strategies. Furthermore, since platinum complexes demonstrate high affinity for nucleophilic sites, various studies have investigated their ability to trigger interactions at the molecular level by binding to various intracellular non DNA components that constitute as many potential targets of their cytotoxicity or resistance. This rationale is schematized in Figure 3 with the example of cisplatin whose participation in DNA adducts accounts for only about $10 \%$ of the whole amount of cisplatin covalently bound to biomolecules within cells $[4,13,17]$. Therefore, the proper significance of the multifactorial mechanisms that mediate cytotoxicity in a highly concerted way both at the cellular and molecular levels should be reconsidered with the perspective of giving traditional drugs a new impetus [5,75].

\section{Innovative synergies}

The combination of alkylating agents or platinum derivatives with relevant therapeutic strategies capable of promoting a synergistic effect and therefore potentiating anticancer activity is of paramount interest in the treatment of solid tumors, as illustrated in Figure 2-(b) with the example of cisplatin. Inhibition of abundant thiol- and thioether-containing amino acids and proteins for which platinum complexes exhibit high affinity can hamper drug detoxification processes [4]. Based on in vitro assays that demonstrated enhanced cell sensitivity to DNA damage and apoptosis in glioblastoma cell lines exposed to buthionine sulfoximine (BSO) beforehand, a significant inhibition of the tumor growth was achieved in animal groups treated with BSO in combination with either temozolomide or cisplatin compared to animal groups treated with each of these drugs independently. Thanks to a putative synergistic effect, even low doses of anticancer agents were sufficient to achieve substantial outcomes while preventing from severe side effects. According to these promising results, the authors suggest that the combination of glutathione (GSH) inhibitors with alkylating agents or platinum complexes may improve the clinical outcome in brain cancer 
patients [76,77]. Conversely, advantage can be taken of the elevated levels of GSH in resistant cancer cells to specifically damage them [6,78]. Bio-mimicking molecules can also be synthesized to supersede their bio-analogs within the organism. Methylation of the gene's promoter of 6-O-methylguanine-DNA methyltransferase (MGMT), a common feature in glioblastoma diagnosed patients, is of good prognosis since it improves cell sensitivity to temozolomide and results in an increase in median survival [21-23]. O6-benzylguanine, a structural analog of O6-methylguanine, is able to divert and irreversibly inactivate the MGMT enzyme, preventing it from repairing DNA adducts induced by temozolomide. Such synergistic combination is expected to lead to the restoration of tumor sensitivity and to maximize drug cytotoxicity. Despite promising preliminary results, the efficacy of this strategy was limited in clinical practice by severe side effects attributed to the inactivation of the MGMT enzyme also in normal tissues [79,80]. Epigenetic modulations that may alter the DNA repair machinery can play a role in circumventing drug resistance too $[6,13,24]$. DNA demethylating agents are able to reverse hypermethylation of genes involved in the DNA mismatch repair (MMR) pathway whose alteration participates in cell resistance to platinum compounds and is of bad prognosis for patients with ovarian carcinoma. A phase II clinical trial in patients with platinum-resistant ovarian carcinoma supported impairment of gene methylation by low-dose decitabine administration and subsequent alteration of the MMR pathway to restore sensitivity to carboplatin, resulting in high response rates and extended progression-free survival [81]. The expression of a panel of genes involved in cell sensitivity or resistance mechanisms and the molecular pathways below may also be modulated to reverse drug resistance and reach a synergistic effect through miRNA that play a key role in cellular development but also in oncogenesis, cancer progression and drug resistance [82-85].

\section{Development of smart nanocarriers}

Nanotechnologies may offer tremendous opportunities in the field of medicine due to their size and versatility of structure, as described with the example of cisplatin in Figure 2(c). Various drug delivery systems (DDS) have been engineered to locally deliver their bioactive cargo, thus concentrating drug efficacy at the tumor site while preventing from systemic toxicity [86]. Indeed, DDS have been described to passively target tumor cells through the enhanced permeability and retention effect (EPR) [87,88]. Although controversial, this paradigm has given rise to the development of various DDS, including for vectorization of platinum derivatives [70,88-93]. Interestingly, active targeting can be achieved by functionalizing nanocarriers with various ligands that specifically bind to 
receptors overexpressed on the surface of cancer cells such as folate or epidermal growth factor $[87,94,95]$. DDS can also be engineered to specifically reroute a drug to targets whose impairment will trigger a cell signaling cascade likely to entail apoptotic cell death [96]. Designing adaptive systems sensitive to micro-environmental changes, namely environment( $\mathrm{pH}$ [97], enzyme and reductive environment [98]) responsive DDS, further allows for specific targeting and triggered drug release. A controlled release of the drug over time and the subsequent modulation of its pharmacokinetic profile may improve its therapeutic benefit $[45,88,99-104]$.

Although DDS are of high interest to extend the drug lifetime in the general circulation and protect it from deactivation until it reaches its target, alternative routes have been investigated to circumvent physiological barriers. In animal models, the local infusion of liposomes [105], nanoparticles [70,106] or polymeric micelles [56] by CED within the brain parenchyma was reported to substantially enhance the distribution volume of the system in comparison with the free drug, as well as to reduce toxicity and prolong half-life $[45,105,107]$.

Endocytosis has been extensively described as the key mechanism of DDS cellular uptake $[89,95,97,102,108-110]$. Protected from deactivation by the plasma membrane vesicle, quanta of active molecules are conveyed from early endosomes to late endosomes and lysosomes, like a "Trojan horse”, favoring drug release in close vicinity of the nuclear and subsequently promoting interactions with DNA $[91,94,95,110]$. As such, Lipoplatin ${ }^{\mathrm{TM}}$, a liposomal formulation of cisplatin, was reported to bypass membrane transporters and subsequent intracellular trafficking by direct fusion with the cell membrane [94,111].

Thanks to the reduced systemic toxicity that goes along with nanovectorization, new effective drug combinations may be considered. Furthermore, the resort to agents modulating drug resistance mechanisms is of particular interest to enhance cell sensitivity to chemotherapy. Poloxamers have been reported to accumulate within resistant cancer cells and intracellular organelles from where they alter metabolic processes involved in drug efflux and detoxification [94,112]. Similarly, micelles loaded with a Pt(IV) prodrug based on an ethacrynic acid backbone achieved substantial reversal of cisplatin resistance owed to effective GST inhibition, leading to tumor necrosis in vivo [103]. Co-delivery of platinum derivatives and miRNA whose involvement in tumorigenesis was specifically identified could also enable to impede tumor cell proliferation and invasiveness [113]. 
Alternatives that combine nanomedicine and other key therapeutic strategies may have great potential in the clinic too. One example relies on the investigation of the radiosensitizing effect of gold nanoparticles due to high X-ray absorption [114]. The incorporation of high Z platinum compounds into various DDS also potentiate drug efficacy in synergy with radiation therapy [31]. Surface-functionalization of DDS with radiopharmaceutics could further allow

352

353 for targeted molecular nuclear medicine, providing nanosystems with an additional imaging modality. This way towards "theranostics" may be a promising application of DDS in the near future.

From the perspective of personalized medicine, multifunctional nanoplatforms may enable to gather large amount of information relevant to patient care [115]. Therefore, combinatorial systems have been developed to allow for real-time monitoring of the treatment efficacy. Some of these systems require a specific stimulus, either physical (light or heat) or chemical (hypoxic conditions or oxidative stress), to release their pharmaceutically active payload $[99,116,117]$. The therapeutic benefit of such tunable nanosystems is improved by real-time monitoring of their biodistribution within the organism together with the evaluation of patient early response to the treatment [118]. As such, the rise of various DDS with integrated smart functions has already pushed the frontiers of science by making it possible to develop hybrid systems that are able not only to drive the drug to its target but also to monitor its impact, or even intensify it.

\section{Concluding remarks}

Owing to their broad anticancer spectrum, alkylating agents and platinum derivatives are key in the management of solid tumors. Still, they suffer from acute systemic toxicity, sub-optimal treatment schedule, intrinsic or acquired resistance and inadequate routing both at the tissue and cellular levels. In this context, this review envisions promising alternatives to the conventional use of alkylating agents and platinum derivatives in clinical practice, including their administration by appropriate routes depending on the tumor location, an optimized subcellular rerouting, synergistic strategies, and the development of an arsenal of smart nanocarriers. Driven by the necessity to rethink their use through rather simple potentiating therapeutic strategies relevant to the daily needs and clinical practice - instead of developing plenty of new drugs that would quickly face the same issues in terms of limited 
therapeutic index (see Outstanding Questions), we do believe that these old molecules have great promise for future applications in the management of solid tumors.

\section{Acknowledgements}

This work was supported by the "Institut National de la Santé et de la Recherche Médicale" (INSERM), by the University of Angers (Angers, France) and by the University of Liege (Liege, Belgium). It is also related to the LabEx IRON “Innovative Radiopharmaceuticals in Oncology and Neurology" as part of the French government "Investissements d'Avenir" program, to the INCa (Institut National du Cancer) MARENGO consortium "MicroRNA agonist and antagonist Nanomedicines for GliOblastoma treatment: from molecular programmation to preclinical validation through the PL-BIO 2014-2020 grant and to the MuMoFRaT project "Multi-scale Modeling \& simulation of the response to hypoFractionated Radiotherapy or repeated molecular radiation Therapies" supported by "La Région Pays-de-la-Loire” and by the Cancéropôle Grand-Ouest (tumor targeting and radiotherapy network). Hélène Lajous was a Ph.D. student involved in the Erasmus Mundus Joint Doctorate program for Nanomedicine and pharmaceutical innovation (EMJD NanoFar) and received a fellowship from “La Région Pays-de-la-Loire”.

\section{Disclosures}

There are no conflicts of interest to declare.

\section{References}

1 Hanahan, D. and Weinberg, R.A. (2011) Hallmarks of cancer: The next generation. Cell 144, 646-74

2 Ye, W. (2016) The complexity of translating anti-angiogenesis therapy from basic science to the clinic. Dev. Cell 37, 114-25

3 Yang, W.-H. et al. (2017) Revision of the concept of anti-angiogenesis and its applications in tumor treatment. Chronic Dis. Transl. Med. 3, 33-40

4 Mezencev, R. (2015) Interactions of cisplatin with non-DNA targets and their influence on anticancer activity and drug toxicity: The complex world of the platinum complex. Curr. Cancer Drug Targets 14, 794-816 
5 Gibson, D. (2019) Multi-action Pt(IV) anticancer agents; do we understand how they 406 work? J. Inorg. Biochem. 191, 77-84

4076 Kelland, L. (2007) The resurgence of platinum-based cancer chemotherapy. Nat. Rev. Cancer 7, 573-84

Johnstone, T.C. et al. (2015) Third row transition metals for the treatment of cancer. Philos. Trans. R. Soc. A Math. Phys. Eng. Sci. 373, 20140185

Wishart, D.S. et al. (2018) DrugBank 5.0: A major update to the DrugBank database for 2018. Nucleic Acids Res. 46, D1074-D1082

9 Yang, F. et al. (2014) Doxorubicin, DNA torsion, and chromatin dynamics. Biochim. Biophys. Acta 1845, 84-9

Babu, N.J. et al. (2013) Temozolomide hydrochloride dihydrate. CrystEngComm 15, $666-71$

Siddik, Z.H. (2002) Mechanisms of action of cancer chemotherapeutic agents: DNAinteractive alkylating agents and antitumour platinum-based drugs. In The Cancer Handbook 1st Edition (Alison, M. R., ed), John Wiley \& Sons, Ltd

Corte-Rodríguez, M. et al. (2015) Quantitative evaluation of cellular uptake, DNA incorporation and adduct formation in cisplatin sensitive and resistant cell lines: Comparison of different Pt-containing drugs. Biochem. Pharmacol. 98, 69-77

13 Shen, D.-W. et al. (2012) Cisplatin resistance: A cellular self-defense mechanism resulting from multiple epigenetic and genetic changes. Pharmacol. Rev. 64, 706-21

Dasari, S. and Tchounwou, P.B. (2014) Cisplatin in cancer therapy: Molecular mechanisms of action. Eur. J. Pharmacol. 740, 364-78

15 Stewart, D.J. (2007) Mechanisms of resistance to cisplatin and carboplatin. Crit. Rev. Oncol. Hematol. 63, 12-31

Garrido, N. et al. (2008) Cisplatin-mediated impairment of mitochondrial DNA metabolism inversely correlates with glutathione levels. Biochem. J. 414, 93-102

Galluzzi, L. et al. (2014) Systems biology of cisplatin resistance: Past, present and future. Cell Death Dis. 5, e1257-18 Serendipity and chemical biology. Chem. Biol. 20, 648-59

Amable, L. (2016) Cisplatin resistance and opportunities for precision medicine. Pharmacol. Res. 106, 27-36

Fennell, D.A. et al. (2016) Cisplatin in the modern era: The backbone of first-line chemotherapy for non-small cell lung cancer. Cancer Treat. Rev. 44, 42-50 
21 Ostrom, Q. et al. (2013) Gene markers in brain tumors: What the epileptologist should know. Epilepsia 54, 25-9

22 Van Thuijl, H.F. et al. (2015) Evolution of DNA repair defects during malignant progression of low-grade gliomas after temozolomide treatment. Acta Neuropathol. 129, 597-607

23 Hegi, M.E. et al. (2005) MGMT gene silencing and benefit from temozolomide in glioblastoma. N. Engl. J. Med. 352, 997-1003

24 O’Grady, S. et al. (2014) The role of DNA repair pathways in cisplatin resistant lung cancer. Cancer Treat. Rev. 40, 1161-70

25 Hoebers, F.J.P. et al. (2008) Cisplatin-DNA adduct formation in patients treated with cisplatin-based chemoradiation: Lack of correlation between normal tissues and primary tumor. Cancer Chemother. Pharmacol. 61, 1075-81

26 Chalmers, A.J. et al. (2009) Cytotoxic effects of temozolomide and radiation are additive- and schedule-dependent. Int. J. Radiat. Oncol. Biol. Phys. 75, 1511-9

27 Eckert, F. et al. (2010) Definitive radiotherapy and single-agent radiosensitizing ifosfamide in patients with localized, irresectable soft tissue sarcoma: A retrospective analysis. Radiat. Oncol. 5, 55

28 Wilson, G.D. et al. (2006) Biologic basis for combining drugs with radiation. Semin. Radiat. Oncol. 16, 2-9

29 Reboul, F.L. (2004) Radiotherapy and chemotherapy in locally advanced non-small cell lung cancer: Preclinical and early clinical data. Hematol. Oncol. Clin. North Am. 18, 41-53

30 Tippayamontri, T. et al. (2012) Synergism in concomitant chemoradiotherapy of cisplatin and oxaliplatin and their liposomal formulation in the human colorectal cancer HCT116 model. Anticancer Res. 32, 4395-404

31 Miladi, I. et al. (2015) Combining ultrasmall gadolinium-based nanoparticles with photon irradiation overcomes radioresistance of head and neck squamous cell carcinoma. Nanomedicine Nanotechnology, Biol. Med. 11, 247-57

32 Tomoda, K. et al. (2015) Examination of gossypol-pluronic micelles as potential radiosensitizers. AAPS J. 17, 1369-75

33 De Felice, F. et al. (2015) Head and neck cancer: Metronomic chemotherapy. BMC Cancer 15, 677

34 Goldberg, R.M. et al. (2004) A randomized controlled trial of fluorouracil plus leucovorin, irinotecan, and oxaliplatin combinations in patients with previously 
untreated metastatic colorectal cancer. J. Clin. Oncol. 22, 23-30

35 Carethers, J.M. (2008) Systemic treatment of advanced colorectal cancer: Tailoring therapy to the tumor. Therap. Adv. Gastroenterol. 1, 33-42

Oldenburg, J. et al. (2013) Testicular seminoma and non-seminoma: ESMO Clinical Practice Guidelines for diagnosis, treatment and follow-up. Ann. Oncol. 24, vi125vi132

37 Bellmunt, J. et al. (2014) Bladder cancer: ESMO Practice Guidelines for diagnosis, treatment and follow-up. Ann. Oncol. 25, iii40-iii48

38 Lordick, F. et al. (2016) Oesophageal cancer: ESMO Clinical Practice Guidelines for diagnosis, treatment and follow-up. Ann. Oncol. 27, v50-v57

39 Novello, S. et al. (2016) Metastatic non-small-cell lung cancer: ESMO Clinical Practice Guidelines for diagnosis, treatment and follow-up. Ann. Oncol. 27, v1-v27

40 Marth, C. et al. (2017) Cervical cancer: ESMO Clinical Practice Guidelines for diagnosis, treatment and follow-up. Ann. Oncol. 28, iv72-iv83

41 Postmus, P.E. et al. (2017) Early and locally advanced non-small-cell lung cancer (NSCLC): ESMO Clinical Practice Guidelines for diagnosis, treatment and follow-up. Ann. Oncol. 28, iv1-iv21

42 Wilson, T. et al. (2014) Glioblastoma multiforme: State of the art and future therapeutics. Surg. Neurol. Int. 5, 132138

43 Lombardi, M.Y. and Assem, M. (2017) Glioblastoma genomics: A very complicated story. In Glioblastoma pp. 3-25, Codon Publications

44 Laquintana, V. et al. (2009) New strategies to deliver anticancer drugs to brain tumors. Expert Opin. Drug Deliv. 6, 1017-32

45 Zhou, J. et al. (2012) Novel delivery strategies for glioblastoma. Cancer J. 18, 89-9

46 Fernandes, C. et al. (2017) Current standards of care in glioblastoma therapy. In Glioblastoma pp. 197-241, Codon Publications

47 Hanif, F. et al. (2017) Glioblastoma multiforme: A review of its epidemiology and pathogenesis through clinical presentation and treatment. Asian Pacific J. Cancer Prev. 18, 3-9

48 Arshad, A. et al. (2015) Convection-enhanced delivery of carboplatin PLGA nanoparticles for the treatment of glioblastoma. PLoS One 10, e0132266

49 Garg, T. et al. (2015) Current strategies for targeted delivery of bio-active drug molecules in the treatment of brain tumor. J. Drug Target. 23, 865-87

50 Vogelbaum, M.A. and Aghi, M.K. (2015) Convection-enhanced delivery for the 
treatment of glioblastoma. Neuro. Oncol. 17, ii3-ii8

51 Cheff, D.M. and Hall, M.D. (2017) A drug of such damned nature.1 Challenges and opportunities in translational platinum drug research. J. Med. Chem. 60, 4517-32

52 Shi, M. et al. (2015) Convection-enhancement delivery of platinum-based drugs and Lipoplatin $^{\mathrm{TM}}$ to optimize the concomitant effect with radiotherapy in F98 glioma rat model. Invest. New Drugs 33, 555-63

53 Vogelbaum, M.A. (2007) Convection enhanced delivery for treating brain tumors and selected neurological disorders: Symposium review. J. Neurooncol. 83, 97-109

54 White, E. et al. (2012) A phase I trial of carboplatin administered by convectionenhanced delivery to patients with recurrent/progressive glioblastoma multiforme. Contemp. Clin. Trials 33, 320-31

55 Siegal, T. (2013) Change clinical practice for brain tumor. Neuro. Oncol. 15, 656-69

56 Zhang, R. et al. (2016) Convection-enhanced delivery of SN-38-loaded polymeric micelles (NK012) enables consistent distribution of SN-38 and is effective against rodent intracranial brain tumor models. Drug Deliv. 23, 2780-6

57 Yang, W. et al. (2011) Convection-enhanced delivery of carboplatin in combination with radiotherapy for the treatment of brain tumors. J Neurooncol 101, 379-90

58 Jaaback, K. et al. (2016) Intraperitoneal chemotherapy for the initial management of primary epithelial ovarian cancer (Review). In Cochrane Database of Systematic Reviews pp. CD005340, John Wiley \& Sons, Ltd

59 Jandial, D.A. et al. (2017) A phase I pharmacokinetic study of intraperitoneal bortezomib and carboplatin in patients with persistent or recurrent ovarian cancer: An NRG Oncology/Gynecologic Oncology Group study. Gynecol. Oncol. 145, 236-42

60 Scharovsky, O.G. et al. (2009) Metronomic chemotherapy: Changing the paradigm that more is better. Curr. Oncol. 16, 7-15

61 Zhidkov, N. et al. (2013) Continuous intraperitoneal carboplatin delivery for the treatment of late-stage ovarian cancer. Mol. Pharm. 10, 3315-22

$62 \mathrm{Su}, \mathrm{W} . \mathrm{H}$. et al. (2012) Metronomic therapy for gynecologic cancers. Taiwan. J. Obstet. Gynecol. 51, 167-78

63 Ye, H. et al. (2015) Sustained, low-dose intraperitoneal cisplatin improves treatment outcome in ovarian cancer mouse models. J. Control. Release 220, 358-67

64 Pasquier, E. et al. (2010) Metronomic chemotherapy: New rationale for new directions. Nat. Rev. Clin. Oncol. 7, 455-65

65 Kubota, H. et al. (2017) Feasibility of metronomic chemotherapy with tegafur-uracil, 
cisplatin, and dexamethasone for docetaxel-refractory prostate cancer. J Rural Med 12, $112-9$

54366 Patil, V.M. et al. (2015) A prospective randomized phase II study comparing metronomic chemotherapy with chemotherapy (single agent cisplatin), in patients with metastatic, relapsed or inoperable squamous cell carcinoma of head and neck. Oral Oncol. 51, 279-86

67 Specenier, P. and Vermorken, J.B. (2018) Optimizing treatments for recurrent or 548 metastatic head and neck squamous cell carcinoma. Expert Rev. Anticancer Ther. 18, 901-15

68 Correale, P. et al. (2011) Phase II trial of bevacizumab and dose/dense chemotherapy with cisplatin and metronomic daily oral etoposide in advanced non-small-cell-lung cancer patients. Cancer Biol. Ther. 12, 112-8

69 Tippayamontri, T. et al. (2011) Cellular uptake and cytoplasm/DNA distribution of cisplatin and oxaliplatin and their liposomal formulation in human colorectal cancer cell HCT116. Invest. New Drugs 29, 1321-7

Lajous, H. et al. (2018) Hybrid Gd3+/cisplatin cross-linked polymer nanoparticles enhance platinum accumulation and formation of DNA adducts in glioblastoma cell lines. Biomater. Sci. 6, 2386-409

71 Cullen, K.J. et al. (2007) Mitochondria as a critical target of the chemotherapeutic agent cisplatin in head and neck cancer. J. Bioenerg. Biomembr. 39, 43-50

72 Van Gisbergen, M.W. et al. (2015) How do changes in the mtDNA and mitochondrial dysfunction influence cancer and cancer therapy? Challenges, opportunities and models. Mutat. Res. 764, 16-30

73 Fonseca, S.B. et al. (2011) Rerouting chlorambucil to mitochondria combats drug deactivation and resistance in cancer cells. Chem. Biol. 18, 445-53

74 Wisnovsky, S.P. et al. (2013) Targeting mitochondrial DNA with a platinum-based anticancer agent. Chem. Biol. 20, 1323-8

Gibson, D. (2016) Platinum(IV) anticancer prodrugs - hypotheses and facts. Dalton Trans. 45, 12983-91

Rocha, C.R.R. et al. (2014) Glutathione depletion sensitizes cisplatin- and temozolomide-resistant glioma cells in vitro and in vivo. Cell Death Dis. 5, e1505 Villablanca, J.G. et al. (2016) A phase I new approaches to neuroblastoma therapy study of buthionine sulfoximine and melphalan with autologous stem cells for recurrent/refractory high-risk neuroblastoma. Pediatr. Blood Cancer 63, 1349-56 
57578 Vergote, I. et al. (2009) Phase 3 randomised study of canfosfamide (Telcyta®, TLK286) versus pegylated liposomal doxorubicin or topotecan as third-line therapy in patients with platinum-refractory or -resistant ovarian cancer. Eur. J. Cancer 45, 232432

Kaina, B. et al. (2010) Targeting O6-methylguanine-DNA methyltransferase with specific inhibitors as a strategy in cancer therapy. Cell. Mol. Life Sci. 67, 3663-81

Warren, K.E. et al. (2012) A phase II study of O6-benzylguanine and temozolomide in pediatric patients with recurrent or progressive high-grade gliomas and brainstem gliomas: A Pediatric Brain Tumor Consortium study. J. Neurooncol. 106, 643-9

Matei, D. et al. (2012) Epigenetic resensitization to platinum in ovarian cancer. Cancer Res. 72, 2197-205

Garzon, R. et al. (2009) MicroRNAs in cancer. Annu. Rev. Med. 60, 167-79

Visone, R. and Croce, C.M. (2009) MiRNAs and cancer. Am. J. Pathol. 174, 1131-8

Chen, W. et al. (2014) MiR-136 targets E2F1 to reverse cisplatin chemosensitivity in glioma cells. J. Neurooncol. 120, 43-53

85 Chen, X. et al. (2015) MiR-873 acts as a novel sensitizer of glioma cells to cisplatin by targeting Bcl-2. Int. J. Oncol. 47, 1603-11

Ventola, C.L. (2017) Progress in nanomedicine: Approved and investigational nanodrugs. Pharm. Ther. 42, 742-55

Peer, D. et al. (2007) Nanocarriers as an emerging platform for cancer therapy. Nat. Nanotechnol. 2, 751-60

88 Harper, B.W. et al. (2010) Advances in platinum chemotherapeutics. Chem. - A Eur. J. 16, 7064-77

89 Cabral, H. and Kataoka, K. (2014) Progress of drug-loaded polymeric micelles into 600 clinical studies. J. Control. Release 190, 465-76

601

90 Li, J. et al. (2014) Complex of cisplatin with biocompatible poly(ethylene glycol) with pendant carboxyl groups for the effective treatment of liver cancer. J. Appl. Polym. Sci. 131, 9246-54

Duan, X. et al. (2015) Polymeric micelle-mediated delivery of DNA-targeting 606 organometallic complexes for resistant ovarian cancer treatment. Small 11, 3962-72 Johnstone, T.C. et al. (2016) The next generation of platinum drugs: Targeted Pt(II) agents, nanoparticle delivery, and Pt(IV) prodrugs. Chem. Rev. 116, 3436-86

Parhizkar, M. et al. (2016) Electrohydrodynamic encapsulation of cisplatin in poly (lactic-co-glycolic acid) nanoparticles for controlled drug delivery. Nanomedicine 
Nanotechnology, Biol. Med. 12, 1919-29

94 Xue, X. et al. (2013) Nanoscale drug delivery platforms overcome platinum-based resistance in cancer cells due to abnormal membrane protein trafficking. ACS Nano 7, 10452-64

95 Lehner, R. et al. (2013) Intelligent nanomaterials for medicine: Carrier platforms and targeting strategies in the context of clinical application. Nanomedicine Nanotechnology, Biol. Med. 9, 742-57

96 Marrache, S. et al. (2014) Detouring of cisplatin to access mitochondrial genome for overcoming resistance. Proc. Natl. Acad. Sci. 111, 10444-9

97 Sun, X. et al. (2017) A pH-responsive yolk-like nanoplatform for tumor targeted dualmode magnetic resonance imaging and chemotherapy. ACS Nano 11, 7049-59

98 Cajot, S. et al. (2011) Design of reversibly core cross-linked micelles sensitive to reductive environment. J. Control. Release 152, 30-6

99 Nishiyama, N. and Kataoka, K. (2006) Current state, achievements, and future prospects of polymeric micelles as nanocarriers for drug and gene delivery. Pharmacol. Ther. 112, 630-48

100 Oerlemans, C. et al. (2010) Polymeric micelles in anticancer therapy: Targeting, imaging and triggered release. Pharm. Res. 27, 2569-89

101 Stathopoulos, G.P. and Boulikas, T. (2012) Lipoplatin formulation review article. J. Drug Deliv. 2012, 581363

102 Patra, H.K. and Turner, A.P.F. (2014) The potential legacy of cancer nanotechnology: Cellular selection. Trends Biotechnol. 32, 21-31

$103 \mathrm{Li}, \mathrm{S}$. et al. (2017) Overcoming resistance to cisplatin by inhibition of glutathione Stransferases (GSTs) with ethacraplatin micelles in vitro and in vivo. Biomaterials 144, 119-29

104 Xu, S. et al. (2017) Supramolecular cisplatin-vorinostat nanodrug for overcoming drug resistance in cancer synergistic therapy. J. Control. Release 266, 36-46

105 Krauze, M.T. et al. (2005) Real-time visualization and characterization of liposomal delivery into the monkey brain by magnetic resonance imaging. Brain Res. Protoc. 16, 20-6

106 Zhang, C. et al. (2017) Convection enhanced delivery of cisplatin-loaded brain penetrating nanoparticles cures malignant glioma in rats. J. Control. Release 263, 1129

107 Saito, R. et al. (2005) Gadolinium-loaded liposomes allow for real-time magnetic 
resonance imaging of convection-enhanced delivery in the primate brain. Exp. Neurol. 196, 381-9

108 Hillaireau, H. and Couvreur, P. (2009) Nanocarriers' entry into the cell: Relevance to drug delivery. Cell. Mol. Life Sci. 66, 2873-96

109 Bregoli, L. et al. (2016) Nanomedicine applied to translational oncology: A future perspective on cancer treatment. Nanomedicine Nanotechnology, Biol. Med. 12, 81103

110 Cai, Y. et al. (2017) Supramolecular “Trojan Horse” for nuclear delivery of dual anticancer drugs. J. Am. Chem. Soc. 139, 2876-9

111 Boulikas, T. (2009) Clinical overview on Lipoplatin $^{\mathrm{TM}}$ : A successful liposomal formulation of cisplatin. Expert Opin. Investig. Drugs 18, 1197-218

112 Exner, A.A. et al. (2005) Enhancement of carboplatin toxicity by Pluronic block copolymers. J. Control. Release 106, 188-97

113 Yang, T. et al. (2016) Anti-tumor efficiency of lipid-coated cisplatin nanoparticles coloaded with microRNA-375. Theranostics 6, 142-54

114 Hainfeld, J.F. et al. (2013) Gold nanoparticle imaging and radiotherapy of brain tumors in mice. Nanomedicine 8, 1601-9

115 McCarthy, J.R. and Weissleder, R. (2008) Multifunctional magnetic nanoparticles for targeted imaging and therapy. Adv. Drug Deliv. Rev. 60, 1241-51

116 Zhu, H. et al. (2012) Responsive fluorescent Bi2O3@PVA hybrid nanogels for temperature-sensing, dual-modal imaging, and drug delivery. Biomaterials 33, 305869

117 Cao, Y. et al. (2015) Photo-triggered fluorescent theranostic prodrugs as DNA alkylating agents for mechlorethamine release and spatiotemporal monitoring. Org. Biomol. Chem. 13, 6742-8

118 Yuan, Y. et al. (2014) Targeted theranostic platinum(IV) prodrug with a built-in aggregation-induced emission light-up apoptosis sensor for noninvasive early evaluation of its therapeutic responses in situ. J. Am. Chem. Soc. 136, 2546-54

119 Choi, Y.M. et al. (2015) Mechanism of cisplatin-induced cytotoxicity is correlated to impaired metabolism due to mitochondrial ROS generation. PLoS One 10, e0135083

120 Mandal, R. et al. (2006) Mass spectrometry study of hemoglobin-oxaliplatin complexes in colorectal cancer patients and potential association with chemotherapeutic responses. Rapid Commun. Mass Spectrom. 20, 2533-8 


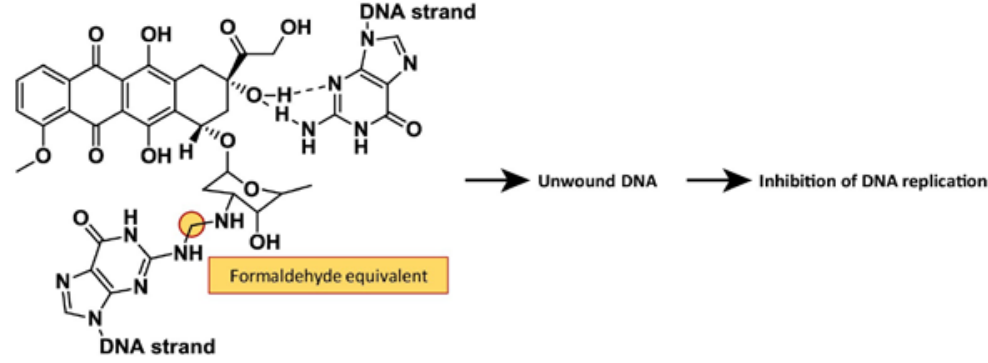

(A)

Guanine on DNA strand (1)

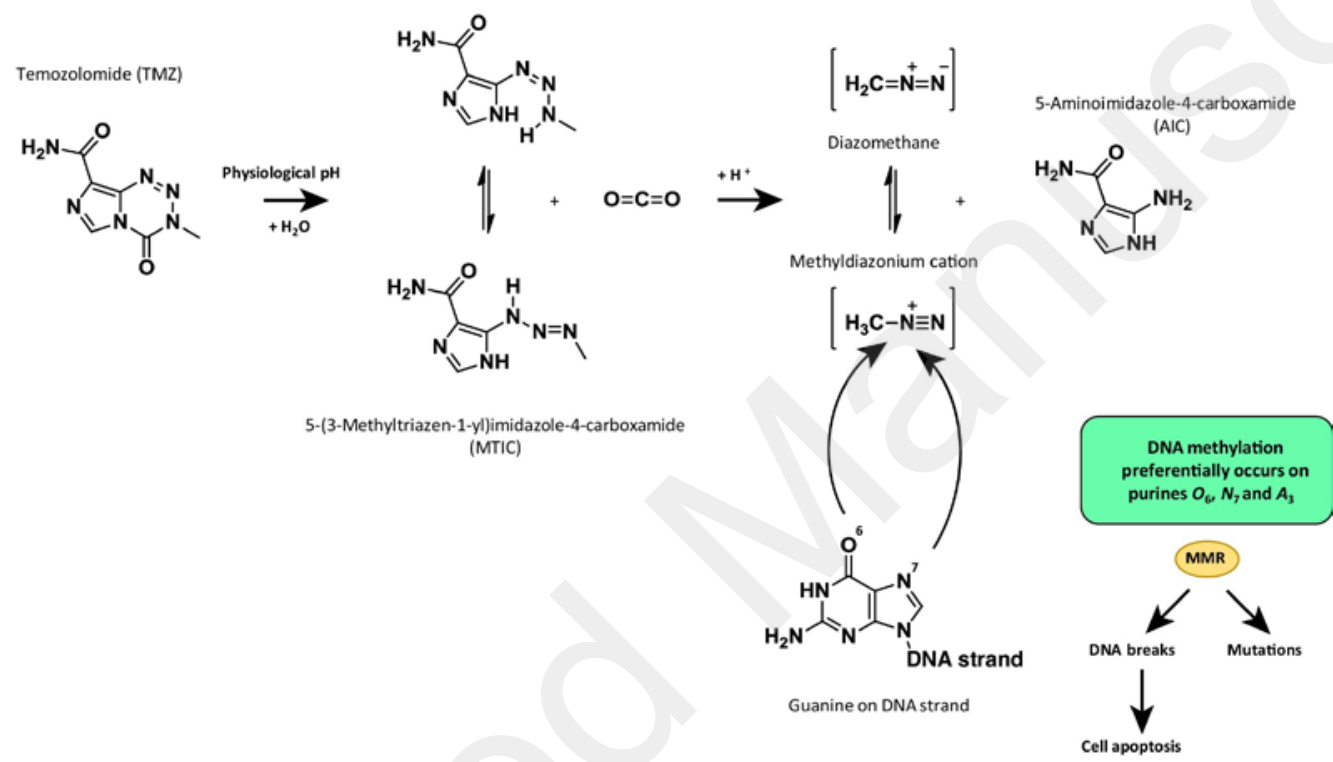

(B)

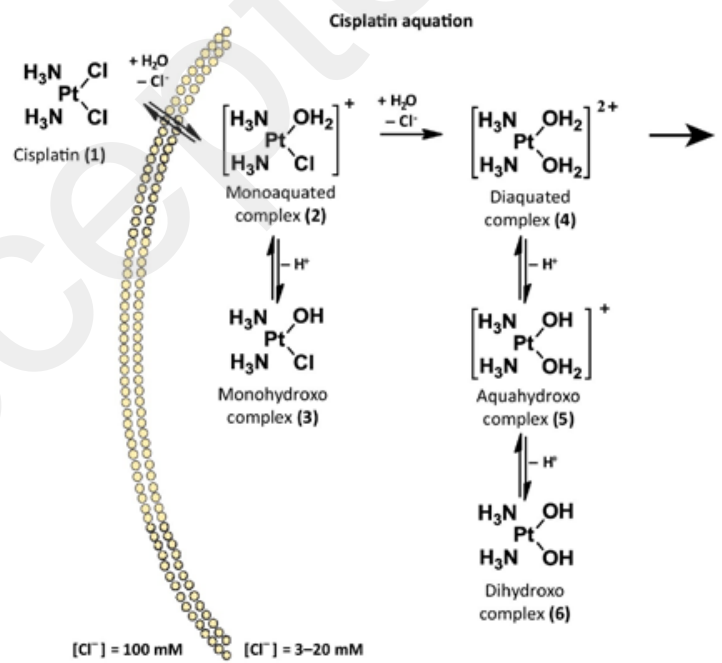

(C)
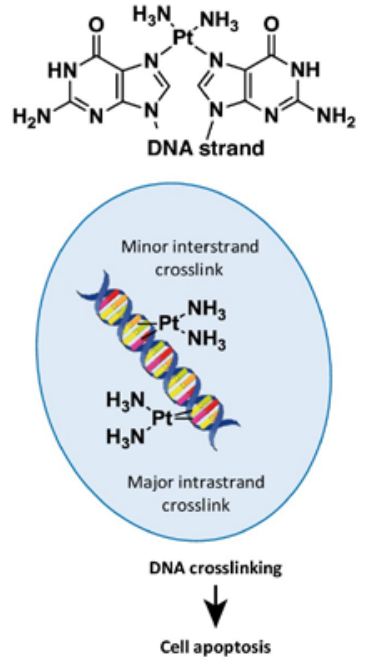

Trends in Pharmacological Sciences 
680

681

682

683

684

685

686

687

688

689

690

691

692

693

694

695

696

697

698

699

Figure 1. Various mechanisms of action of DNA targeting agents. (a) Intercalation of doxorubicin between DNA strands. Doxorubicin forms with guanine a covalent bond (formaldehyde equivalent) on one DNA strand and hydrogen bonds on the opposite strand to stabilize the structure. Consequently, DNA stays unwound and replication becomes impossible. Interactions with DNA preferentially occur with neighboring GC base pairs [9]. (b) DNA methylation by temozolomide. Temozolomide acts as a prodrug spontaneously hydrolyzed at physiological pH in its active metabolite MTIC, subsequently converted to AIC and methyldiazonium [10]. This highly reactive cation methylates purine bases, preferentially $\mathrm{O}_{6}$ and $\mathrm{N}_{7}$ guanines and to a lesser extent $\mathrm{A}_{3}$ adenine, thus inhibiting DNA replication. Excision of $\mathrm{O}_{6}$-methylguanine adducts by MMR enzymes may induce either mutations continuously recovered along replications or DNA single- or double-strand breaks responsible for cell apoptosis [43]. (c) DNA complexation with cisplatin. Cisplatin (1) requires the substitution of at least one chloride group by water for its activation, a process called aquation. This hydrolysis automatically occurs once cisplatin is internalized because of the small intracellular chloride concentration. Reactivity of Pt(II) complexes, (4) > (2),(5) > (1),(3) >> (6), is determined by the ability of every ligand to be substituted by a nucleophile [4]. Active Pt(II) species complex with nucleophilic intracellular ligands: $\mathrm{N}_{7}$-sites of purine DNA or RNA bases, mainly guanine and to a lesser extent adenine, and nucleophilic sites on several proteins [7,12]. Guanine intrastrand cross-linking with cisplatin impedes DNA replication and transcription [6,11]. 


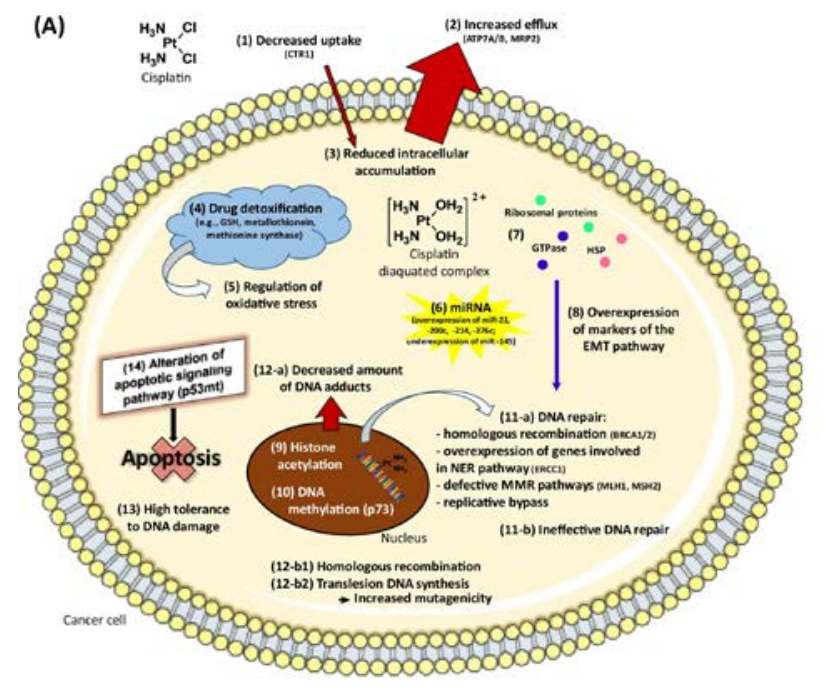

(B)
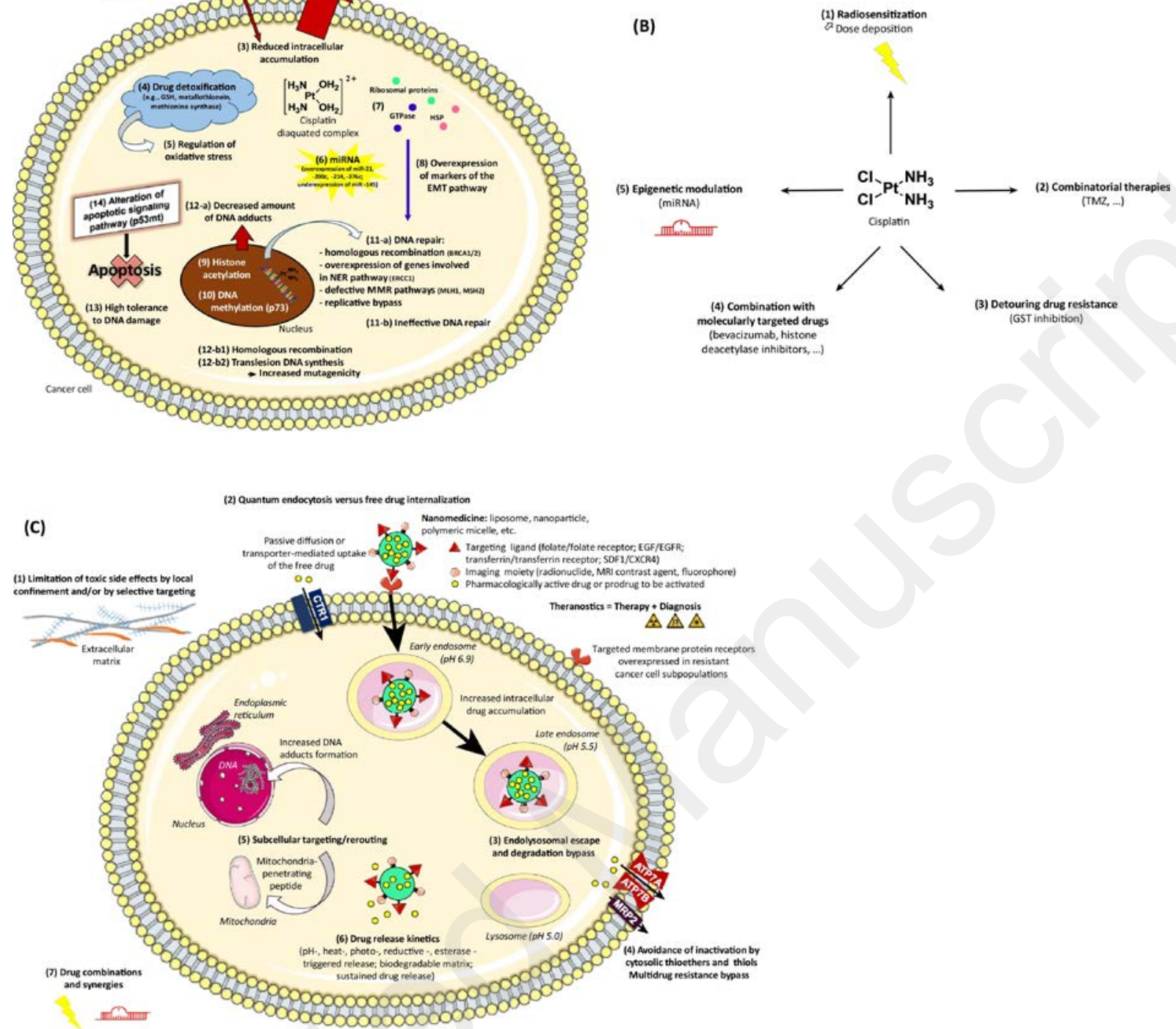

Figure 2, Key Figure. Strategies to overcome cellular resistance and enhance the therapeutic index of a drug: The example of cisplatin. (a) Cellular resistance mechanisms to cisplatin. (1) Impaired influx through altered transported-mediated uptake (CTR1) [13,14], or conversely (2) active efflux outside the cell (ATP7A/B, MRP2) [6] are responsible for (3) a reduced total intracellular accumulation of the drug [12]. Cisplatin efflux from the nucleus back to the cytoplasm also reduces drug distribution to nuclear DNA [15]. (4) The abundance within the cytosol of thiol- and thioether-containing amino acids and proteins for which cisplatin exhibits high affinity is responsible for detoxification processes that lead to drug sequestration and inactivation $[4,16,17,73]$. Besides, glutathione may quench Pt-DNA monoadducts before their conversion into cytotoxic DNA cross-links and (5) reduce cisplatin-induced oxidative stress within cells $[15,16]$. To overcome drug cytotoxicity, tumor cells trigger an overall abnormal phenotype by silencing or activating multiple genes notably 
involved in (6) the modulation of the expression of miRNA, (7) of GTPases, ribosomal and heat shock proteins (HSP), in (8) the overexpression of markers of the epithelial to mesenchymal transition (EMT) pathway, in (9) histone acetylation, (10) aberrant DNA methylation, (11) DNA-damage repair and (14) apoptotic signaling pathways [13]. (11-a) DNA cross-linking recognition and reparation mechanisms allow for (12-a) a decreased amount of DNA adducts, whereas (11-b) ineffective DNA repair leads to (12-b1) homologous recombination or (12-b2) translesion DNA synthesis that further results in genome instability and recurrence of aggressive and resistant tumor cells [16]. Among other indications, cisplatin provided a breakthrough in the management of testis cancer attributed to an intrinsic cellular hypersensitivity together with a reduced ability to repair DNA adducts through the nucleotide excision repair (NER) pathway [6]. Other molecular pathways are also involved in the efficacy/toxicity of platinum-based regimen $[13,15,17,24]$. (13) An enhanced tolerance to DNA damage and (14) the alteration of the apoptotic signaling pathway, especially p53 mutation (p53mt), result in cell escape from apoptosis and acquired resistance [6,15]. (b) Innovative synergies capable of detouring drug resistance mechanisms. (1) Cisplatin is conventionally used in combination with radiotherapy (RT) in the treatment of various solid tumors as it enhances dose deposition [25,28,29]. RT can increase the cellular uptake of cisplatin and promote the activation of toxic Pt(II) complexes. Conversely, cisplatin may stop the cell cycle and inhibit the molecular repair machinery that tackles radiation-induced DNA damage [28]. (2) Interestingly, cisplatin was reported to decrease the MGMT activity whose expression counters temozolomide efficacy in glioma treatment [76]. (3) An alternative to reverse resistance induced detoxification processes consists in taking advantage of the elevated levels of GSH in resistant cancer cells to specifically damage them $[6,78]$ or to inhibit the glutathione S-transferase (GST) [103]. (4) Combination with histone deacetylase inhibitors prevents histones from binding to DNA, leaving it more accessible to alkylation/complexation [104]. (5) Sensitivity can also be restored through epigenetic modulations involving miRNAs for permissive or synergistic effects [82-85]. (c) Advantages of cisplatin nanovectorization over traditional regimens. Multifunctional nanocarriers are developed and evaluated towards an optimized drug delivery to tumor cells for (1) locoregional confinement in specific environments and/or for selective targeting of receptors in relation with administration routes and modalities [87,94,95]. They can also be engineered by using radionuclides, MRI contrast agents or fluorophores to assess the patient response in real-time and adjust the treatment [116,118]. (2) Whereas free molecules individually enter the intracellular space by passive diffusion through the membrane or by transporters 
mediation, endocytosis of nanosized DDS enables the internalization of the drug in a quantum form [109]. Nanocarriers have been synthesized to bypass (3) endolysosomal degradation, (4) detoxification processes and drug elimination by multidrug resistance efflux [94]. Besides, tailored nanosystems can mediate (5) the rerouting or subcellular trafficking of the drug to target specific organelles [96]. The high intracellular platinum accumulation favored by the

752

753

754

755

756

757

758 endocytic process is associated with an increased formation of DNA adducts and a markedly enhanced antitumor activity whatever the resistance status of the cells [69,91,97,103,104]. The versatility of structure of smart DDS also allows for (6) a sustained drug release mediated by environmental triggers in the intracellular compartment or in the extracellular space [98,116]. (7) Drug combinations and synergies with alternative approaches such as adjuvant radiation therapy or modulation of the expression of resistance signals through miRNA agonist or antagonist strategies may reinforce the cytotoxicity of nanovectorized cisplatin.

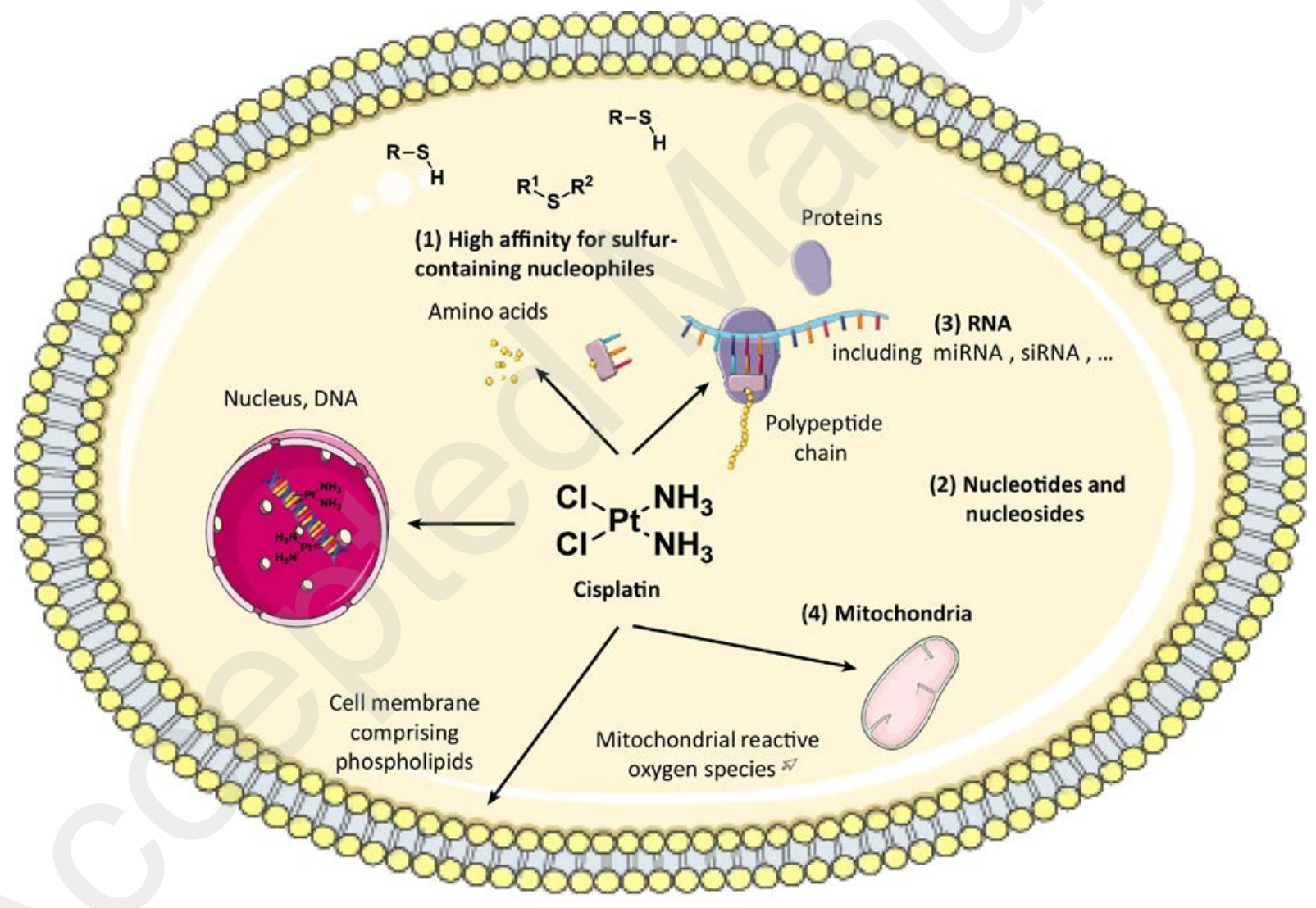

(5) Interactions with various extracellular components

Figure 3. Cisplatin alternative and neglected targets. Cisplatin binds to various intracellular non DNA components that constitute potential targets and factors of efficacy or resistance. (1) Cisplatin interactions with proteins account for most adducts within cells due to high reactivity of thiol and thioether protein constitutive residues and their abundance within 
the cytosol [4]. (2) Nucleotides and nucleosides are characterized by a lower steric hindrance compared to their analogs involved in DNA that may result in easier interactions with $\mathrm{Pt}(\mathrm{II})$ complexes. (3) Kinetics considerations showed faster and higher complexation rates of cisplatin with RNA than DNA in vitro, even though resulting in less stable adducts. Crosslinking with mRNA was reported to inhibit translation in vitro. Interactions with non-coding RNA may impair downstream cellular and molecular processes [4]. (4) Positively charged $\mathrm{Pt}(\mathrm{II})$ activated species were reported to accumulate within negatively charged mitochondria due to electrostatic interactions. There, cisplatin produces a significantly higher amount of adducts with mitochondrial DNA than with nuclear DNA, subsequently impairing response and clinical outcome of cancer patients $[16,72,119]$. Besides, since resistance to cisplatin is partly linked to an extensive repair of Pt-DNA adducts by the NER machinery, rerouting the drug towards mitochondria whose DNA lacks such repair mechanisms may overcome resistance and enhance therapeutic efficacy [96]. (5) Although the amount of Pt(II) complexes with hemoglobin that persist following an oxaliplatin-based treatment was correlated with an increased risk of disease progression in patients with colorectal cancer, the impact of cisplatin interactions with extracellular components has not been reported yet [120]. 


\section{Tables}

782 Table 1. FDA-approved alkylating agents and affiliated compounds for anticancer therapy ${ }^{\mathrm{a}}$.

783 Marketing authorization and clinical practice guidelines are likely to evolve over time and

784 depending on the country.

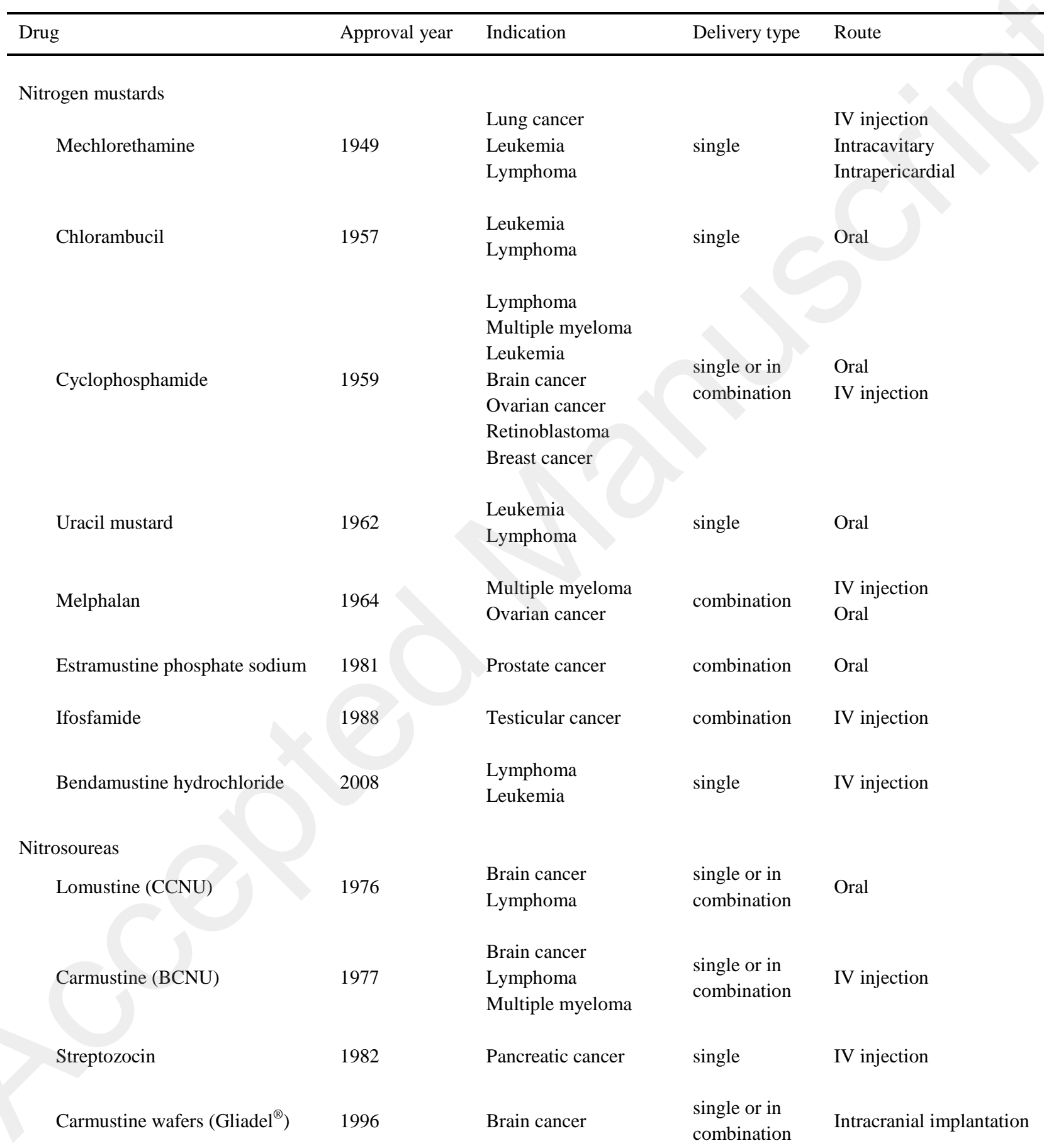




\begin{tabular}{|c|c|c|c|c|}
\hline Drug & Approval year & Indication & Delivery type & Route \\
\hline \multicolumn{5}{|l|}{ Platinum complexes } \\
\hline Cisplatin & 1978 & $\begin{array}{l}\text { Testicular cancer } \\
\text { Ovarian cancer } \\
\text { Bladder cancer }\end{array}$ & $\begin{array}{l}\text { single or in } \\
\text { combination }\end{array}$ & IV injection \\
\hline Carboplatin & 1989 & Ovarian cancer & $\begin{array}{l}\text { single or in } \\
\text { combination }\end{array}$ & IV injection \\
\hline Oxaliplatin & 2004 & $\begin{array}{l}\text { Colon cancer } \\
\text { Colorectal cancer }\end{array}$ & combination & IV injection \\
\hline \multicolumn{5}{|l|}{ Others } \\
\hline Busulfan & 1954 & Leukemia & combination & $\begin{array}{l}\text { Oral } \\
\text { IV injection }\end{array}$ \\
\hline Thiotepa & 1959 & $\begin{array}{l}\text { Breast cancer } \\
\text { Ovarian cancer } \\
\text { Bladder cancer }\end{array}$ & single & $\begin{array}{l}\text { IV injection } \\
\text { Intravesical instillation }\end{array}$ \\
\hline Pipobroman & 1966 & Leukemia & single & Oral \\
\hline Procarbazine hydrochloride & 1969 & Lymphoma & combination & Oral \\
\hline Mitomycin C & 1974 & $\begin{array}{l}\text { Stomach cancer } \\
\text { Pancreatic cancer } \\
\text { Bladder cancer }\end{array}$ & $\begin{array}{l}\text { single or in } \\
\text { combination }\end{array}$ & IV injection \\
\hline Dacarbazine & 1975 & $\begin{array}{l}\text { Melanoma } \\
\text { Lymphoma }\end{array}$ & $\begin{array}{l}\text { single or in } \\
\text { combination }\end{array}$ & IV injection \\
\hline Altretamine & 1990 & Ovarian cancer & single & Oral \\
\hline Temozolomide & 2005 & Brain cancer & $\begin{array}{l}\text { single or in } \\
\text { combination }\end{array}$ & Oral \\
\hline Trabectedin & 2015 & Soft tissue sarcoma & single & IV injection \\
\hline
\end{tabular}

a Abbreviations: IV, intravenous 


\section{Glossary}

787

788

789

790

791

792

793

794

795

796

797

798

799

800

801

802

803

804

805

806

807

808

809

810

811

812

813

814

815

Alkyl group: a univalent group derived from alkanes by removal of a hydrogen atom from any carbon atom, an alkane being an acyclic branched or unbranched hydrocarbon having the general formula $\mathrm{C}_{n} \mathrm{H}_{n+2}$.

Cisplatin, cis-diamminedichloroplatinum(II): a metallic coordination complex with a central platinum atom in a divalent state, two labile chlorine groups and two stable amine ligands located in a cis- configuration. Its ability to inhibit DNA synthesis on E. coli bacterial culture was serendipitously discovered in 1965 by Rosenberg and led to its FDA-approval as an antineoplastic agent in 1978.

Dose deposition: quantifies the concentration of energy absorbed in a tissue following exposure to ionizing radiation. Basically, absorption of X-rays of a given frequency increases with higher $\mathrm{Z}$ atomic number of the penetrated material, which explains the radiosensitizing properties of platinum derivatives.

Drug delivery system (DDS): a formulation or a device that carries a therapeutic compound throughout the body and improves its efficacy while limiting systemic toxicity by controlling the location, the time and the rate of drug release.

Gliadel $^{\circledR}$ : 3.85\% carmustine-loaded polymeric wafers that enable a controlled and sustained drug release. Although controversial, their implantation within the resection bed of operable newly diagnosed glioblastoma patients was approved in 2002 by the FDA as first-line treatment.

Glutathione (GSH): with a concentration of 0.5 to $10 \mathrm{mM}$, this tripeptide is the most abundant thiol within the cell.

Heat shock proteins (HSP): produced by living organisms in response to a stress such as temperature or exposition to heavy metals, overexpressed in cisplatin resistant cells, HSP prevent proteins from impairment.

Maximum tolerated dose (MTD): evaluated in phase I clinical trials, the MTD is the highest dose of a drug or a treatment that does not induce unacceptable side effects.

Metallothioneins: proteins constituted of high amounts of sulfur-rich amino acids, namely cysteine. Exhibiting high affinity to metals, they play a key role in drug detoxification.

MGMT, 6-O-methylguanine-DNA methyltransferase: enzyme involved in the repair of methylated DNA adducts. 
817 microRNA (miRNA): regulatory endogenous non-coding RNAs produced by the genome.

818 p53: tumor suppressor notably involved in cell cycle regulation and apoptotic cell death, its 819 mutation is a common feature in human cancer cells.

820 Poloxamers: amphiphilic block copolymers able to self-assemble into micelles.

821 Targeted therapies: therapeutic strategies that use drugs or other substances to recognize 822 particular entities associated with hallmarks of cancer cells while sparing normal cells. Some 823 targeted therapies work by blocking the action of cancer's specific genes, proteins, or 824 environmental cues that contribute to cancer growth and survival.

825 Temozolomide: small orally available lipophilic molecule of high interest in the treatment of 826 malignant gliomas due to its ability to cross the blood-brain barrier.

827 Theranostics: merger between the words "therapy” and "diagnosis". 